\title{
Extremal Graphs for Estrada Indices
}

\author{
Enide Andrade \\ CIDMA-Center for Research and Development in Mathematics and Applications \\ Department of Mathematics, University of Aveiro, 3810-193, Aveiro, Portugal. \\ Eber Lenes \\ Área de Básicas Exactas, Universidad del Sinú, Cartagena, Colombia \\ Grupo de Investigación Deartica. \\ Exequiel Mallea-Zepeda \\ Departamento de Matemática, Universidad de Tarapacá, Arica, Chile. \\ María Robbiano \\ Departamento de Matemáticas, Universidad Católica del Norte, Av. Angamos 0610 \\ Antofagasta, Chile. \\ Jonnathan Rodríguez Z. \\ Departamento de Matemáticas, Facultad de Ciencias Básicas, Universidad de \\ Antofagasta, Av. Angamos 0601 Antofagasta, Chile.
}

\begin{abstract}
Let $\mathcal{G}$ be a simple undirected connected graph. The signless Laplacian Estrada, Laplacian Estrada and Estrada indices of a graph $\mathcal{G}$ is the sum of the exponentials of the signless Laplacian eigenvalues, Laplacian eigenvalues and eigenvalues of $\mathcal{G}$, respectively. The present work derives an upper bound for the Estrada index of a graph as a function of its chromatic number, in the family of graphs whose color classes have order not less than a fixed positive integer. The graphs for which the upper bound is tight is obtained. Additionally, an upper bound for the Estrada Index of the complement of a graph in the previous family of graphs with two color classes is given. A Nordhaus-Gaddum type inequality for the Laplacian Estrada index when $\mathcal{G}$ is a bipartite graph with color classes of order not less than 2, is presented.
\end{abstract}

\footnotetext{
${ }^{*}$ Corresponding author
} 
Moreover, a sharp upper bound for the Estrada index of the line graph and for the signless Laplacian index of a graph in terms of connectivity is obtained.

Keywords: Estrada index; signless Laplacian Estrada index; Laplacian Estrada index; chromatic number; vertex connectivity; edge connectivity; line graph.

2000 MSC: 05C50, 15A18

\section{Introduction}

This work deals with undirected simple graphs herein called graphs. Given a graph $\mathcal{G}$, we write $V(\mathcal{G})$ and $E(\mathcal{G})$ for the vertex and edge set of $\mathcal{G}$, respectively. Sometimes, after a labeling of the vertices of $\mathcal{G}$, a vertex $v_{i}$ is simply identified by its label $i$ and an edge $v_{i} v_{j}$ can be simply written as $i j$. The degree of a vertex $v_{i}$ is $d\left(v_{i}\right)$ or simply $d_{i}$. The complement of a graph $\mathcal{G}$ is represented by $\overline{\mathcal{G}}$. We will denote the adjacency and diagonal matrix of vertex degrees of a graph $\mathcal{G}$ by $A(\mathcal{G})$ and $D(\mathcal{G})$, respectively. The Laplacian and signless Laplacian matrices of $\mathcal{G}$ are given by $L(\mathcal{G})=D(\mathcal{G})-A(\mathcal{G})$ and $Q(\mathcal{G})=D(\mathcal{G})+A(\mathcal{G})$, respectively.

Since $A(\mathcal{G}), Q(\mathcal{G})$ and $L(\mathcal{G})$ are Hermitian matrices, it follows that they have real spectra. As two distinct labeling of the vertices of $\mathcal{G}$ produce permutationally similar matrices to these matrices, their spectra are independent of the labeling. The eigenvalues of $A(\mathcal{G})$ are the eigenvalues of the graph $\mathcal{G}$. Through this paper, the adjacency, signless Laplacian and Laplacian eigenvalues of a graph $\mathcal{G}$, will be considered by, $\lambda_{1} \geq \ldots \geq \lambda_{n}, q_{1} \geq \ldots \geq q_{n}$ and $\mu_{1} \geq \ldots \geq \mu_{n}$, respectively. The line graph of a graph $\mathcal{G}$ will be denoted by $\mathcal{L}(\mathcal{G})$. Given two vertex disjoint graphs $\mathcal{G}_{1}$ and $\mathcal{G}_{2}$, the join of $\mathcal{G}_{1}$ and $\mathcal{G}_{2}$ is the graph denoted by $\mathcal{G}=\mathcal{G}_{1} \vee \mathcal{G}_{2}$ and the complete $s$-partite graph on $n$ vertices will be denoted by $\mathcal{K}_{n_{1}, \ldots, n_{s}}$, where $n=n_{1}+\ldots+n_{s}$. During the paper the following definitions are considered, the vertex connectivity, or just connectivity of a graph $\mathcal{G}$, is the minimum number of vertices whose deletion disconnects $\mathcal{G}$. An independent set in $\mathcal{G}$ is a set of vertices, no two of which are adjacent. A coloring of a graph $\mathcal{G}$ is an assignment of colors to its vertices so that any pair of connected vertices have distinct colors. The chromatic number $\chi(\mathcal{G})$ of the graph $\mathcal{G}$ is the minimum number of colors among all the possibility of coloring of $\mathcal{G}$. A color class is the set of all vertices of a single color. Clearly, any color class is an independent set. In this paper, the complete graph, the path, the star and the cycle of orders $n$ are denoted by 
$\mathcal{K}_{n}, \mathcal{P}_{n}, \mathcal{S}_{n}$ and $\mathcal{C}_{n}$, respectively. The complete bipartite graph, is denoted by $\mathcal{K}_{p, q}$, where $p$ and $q$ are the cardinalities of its independent sets.

The spectrum of a square matrix $M$ (the multiset of the eigenvalues of $M$ ) will be denoted here by $S p(M)$. Here $|U|$ is the cardinality of the finite set $U$.

\section{The Estrada Indices and some of its applications}

Based on research on geometric properties of biomolecules [? ? ], given a molecular graph $\mathcal{G}$ with eigenvalues $\lambda_{1}, \ldots, \lambda_{n}$, Ernesto Estrada conceived the following expression

$$
E E(\mathcal{G})=\sum_{i=1}^{n} \exp \left(\lambda_{i}\right)
$$

The mathematical significance of the quantity in (??) was recognized short time later [? ] and soon it became known under the name of "Estrada index" [? ] (originally it was called "the sum of the subgraph centralities").

This spectra graph invariant is used in many research and knowledge areas. Concerning weighted graphs, the Estrada index can be used in the research of molecular structures, see [? ? ? ]. Ernesto Estrada was motivated when noted that the folding degree of chains is central to the elucidation of structure-function relationships in proteins. This new index characterize the folding degree of a (protein) chain and it is based on the spectral moments of a matrix representing the dihedral angles of the protein main chain, see [? ]. For graphs without weights the reader should refer to [? ? ] where the authors presented a measure of centrality and bipartivity of certain networks such as the metabolism, social communities, individual proteins and trophic interactions in food networks. A relation between the Estrada Index and an atomic branching can be seen in [? ]. Here the authors gave a graph theoretic measure of extended atomic branching that explains the effect of all atoms in a molecule, giving higher weight to the nearest neighbors. They proved a result that allows the exact calculation of this measure based on the eigenvalues and eigenvectors of the adjacency matrix of the graph representing a molecule.

Eventually, a plethora of investigations of the Estrada index followed, whose details can be found in the surveys [? ? ] and, for instance in the papers [? ? ? ? ? ]. Applications of the Estrada index in Shannon entropy, 
network theory and thermodynamics can be found in [? ], [? ] and [? ? ] , respectively.

Invariants of the form (1) can be conceived for any Hermitian matrix $M$ with spectrum $S p(M)$

$$
E E(M)=\sum_{\alpha_{i} \in S p(M)} \exp \left(\alpha_{i}\right) .
$$

The Laplacian Estrada index [? ? ], was defined as

$$
L E E(\mathcal{G})=\sum_{i=1}^{n} \exp \left(\mu_{i}\right)
$$

For more information on this graph invariant see some of the most cited paper [? ? ? ] and some of the recent papers [? ? ? ] and the references therein. In [?], Zhou and Gutman established lower and upper bounds for $L E E(\mathcal{G})$ in terms of number of vertices, edges and the first Zagreb index. The authors also introduce a nice relation between $L E E$ of bipartite graphs with $n$ vertices and $m$ edges and the $E E$ of its line graph, that is

$$
L E E(\mathcal{G})=n-m+e^{2} E E(\mathcal{L}(\mathcal{G}))
$$

with $e=\exp (1)$. In consequence, in [? ] the authors proved that the path and the star have minimal and maximal $L E E$ among all trees on $n$ vertices, respectively, which showed the use of the Laplacian Estrada index as a measure of branching in alkanes.

The signless Laplacian Estrada index [? ], defined in a similar way,

$$
S L E E(\mathcal{G})=\sum_{i=1}^{n} \exp \left(q_{i}\right),
$$

has attracted particular attention. For some details on this graph invariant see [? ? ]. Note that $S L E E$ and $L E E$ coincide in the case of bipartite graphs. This is an immediate consequence of the fact [?] that the Laplacian and signless Laplacian spectra of bipartite graphs coincide. Since the vast majority of molecular graphs are bipartite, for this type of graphs SLEE doesn't add anything new to the previous study of $L E E$. Therefore, its interest comes for non bipartite graphs. In Chemistry, cases where SLEE 
and $L E E$ differ are the fullerenes, fluoranthenes and other non-alternant conjugated species, see [? ? ? ].

Related with Estrada indices, for instance, Zhu [? ] determined the extremal graphs that maximize the $L E E$ for graphs with given connectivity $k$ or given matching number. Here, we study a similar problem considering the chromatic number and the (vertex and edge) connectivity for the different Estrada indices. After the presentation of the notation and the Estrada indices, this paper is organized as follows. In Section 3, in order to extend a result in [?], a sharp upper bound for the Laplacian Estrada index of graphs in terms of the chromatic number $\chi$, in a family of simple undirected connected graphs whose color classes have order not less than a positive integer $r$, is established. At Section 4 an upper bound for the Estrada Index of the complement of a graph in the previous family of graphs when $\chi=$ 2 , is given. A Nordhaus-Gaddum type inequality for $L E E(\mathcal{G})$ when $\mathcal{G}$ is bipartite with color classes of order not less than 2 is presented. A sharp upper bound for the Estrada index of the line graph of a graph and for the signless Laplacian index of a graph in terms of connectivity is obtained in Section 4 and Section 5, respectively. At Section 6 it is presented a result about graphs with maximal signless Laplacian Estrada index and, at Section 7 some computational experiments are given in order to compare our new upper bounds with real values of the Estrada indices for certain connected graphs. Finally, at Section 8 we present some conclusions summarizing the study done and give some insight for future work.

\section{Laplacian Estrada Index and Chromatic Number}

In this section a result from X. Chen \& Y. Hou, presented in [? ], is extended to a family of a simple undirected connected graphs on $n$ vertices whose color classes have order not less than a fixed positive integer $r$. Let $\mathcal{F}_{n}$ be the family of the simple undirected connected graphs on $n$ vertices. Let

$$
\mathcal{A}_{n}^{\chi}=\left\{\mathcal{G} \in \mathcal{F}_{n}: \chi(\mathcal{G})=\chi\right\} .
$$

Let $\mathcal{B}_{n, \chi}^{r}$ be the family of graphs that belong to the set $\mathcal{A}_{n}^{\chi}$ whose color classes have order not less than $r$. Then, the next result from Chen and Y. Hou, [? ], can be rewritten in the light of this definition.

Proposition 1. [? ] Let $\mathcal{G} \in \mathcal{B}_{n, \chi}^{1}$ where $2 \leq \chi \leq n$ and $n=s \chi+t$ with $0 \leq t<\chi$. 
1. If $n=\chi+t$ with $0 \leq t<\chi$, or $n=2 \chi+t$ with $0 \leq t \leq 3$, or $n=3 \chi$ with $2 \leq \chi \leq 3$, then

$L E E(\mathcal{G}) \leq 1+(\chi-1) \exp (n)+(\chi-t)(s-1) \exp (n-s)+s t \exp (n-s-1)$,

where equality holds in (??) if and only if $\mathcal{G} \cong \mathcal{K}_{\underbrace{s, \ldots, s}_{\chi-t}}^{s, \underbrace{s+1, \ldots, s+1}_{t}}$.

2. If $n=2 \chi+t$ with $4 \leq t<\chi$, or $n=3 \chi$ with $\chi \geq 4$, or $n>3 \chi$, then

$$
L E E(\mathcal{G}) \leq 1+(\chi-1)(\exp (n)+\exp (n-2))+(n-2 \chi+1) \exp (2 \chi-2),
$$

where equality holds in (??) if and only if $\mathcal{G} \cong \mathcal{K}_{2} \underbrace{2, \ldots, 2}_{\chi-1} \underbrace{n-2 \chi+2}_{1}$.

The following corollary is an immediate consequence.

Corollary 2. Let $\mathcal{G} \in \mathcal{B}_{n, \chi}^{2}$ where $2 \leq \chi \leq n$ and $n=s \chi+t$ with $0 \leq t<\chi$.

1. If $n=2 \chi+t$ with $0 \leq t<3$, or $n=3 \chi$ with $2 \leq \chi \leq 3$, then

$L E E(\mathcal{G}) \leq\left\{\begin{array}{l}1+(\chi-1) \exp (n)+(\chi-t) \exp (n-2)+2 t \exp (n-3), \text { if } n=2 \chi+t, \\ 1+(\chi-1) \exp (n)+2(\chi-t) \exp (n-3)+3 t \exp (n-4), \text { if } n=3 \chi\end{array}\right.$

If $n=2 \chi+t$, the equality holds in (??) if and only if $\mathcal{G} \cong \mathcal{K}_{\underbrace{2, \ldots, 2}_{\chi-t}} \underbrace{3, \ldots, 3}_{t}$.

If $n=3 \chi$, the equality holds in (??) if and only if $\underbrace{\mathcal{K}_{3}, \ldots, 3}_{\chi}$.

2. If $n=2 \chi+t$ with $4 \leq t<\chi$, or $n=3 \chi$ with $\chi \geq 4$, or $n>3 \chi$, then

$$
L E E(\mathcal{G}) \leq 1+(\chi-1)(\exp (n)+\exp (n-2))+(n-2 \chi+1) \exp (2 \chi-2),
$$

where equality holds in (??) if and only if $\mathcal{G} \cong \mathcal{K}_{2} \underbrace{2, \ldots, 2}_{\chi-1} \underbrace{n-2 \chi+2}_{1}$.

One of the main goals of this paper is to extend the above results for the set $\mathcal{B}_{n, \chi}^{r}$ with $r \geq 3$. Below we present some preliminary results for the proof of the main theorem of this section.

Lemma 3. Let $X \geq 1$ and $Y \geq 0$. Then

$$
X+Y \leq X \exp (Y)
$$

with equality if and only if $Y=0$. 
Proof. Let $Y \geq 0$ and $X=1+a$ for some $a \geq 0$. It is known that $Y+1 \leq \exp (Y)$ and $a \leq a \exp (Y)$ with equality if and only if $Y=0$. Then $(1+a)+Y \leq(1+a) \exp (Y)$ with equality if and only if $Y=0$. That is, $X+Y \leq X \exp (Y)$ with equality if and only if $Y=0$.

Lemma 4. Let $n_{i} \geq 4$, for $i=1, \ldots, \chi$ and consider the sum

$$
\sum_{i=1}^{\chi}\left(n_{i}-1\right) \exp \left(n-n_{i}\right)
$$

If any pair $\left(n_{i}, n_{j}\right)$ with $i \neq j$ is replaced in (??) by $\left(n_{i}-1, n_{j}+1\right)$, then the sum increases.

Proof. Let $m \geq 8$ and $3 \leq x \leq \frac{m}{2}$. By Lemma ??, we have

$$
\begin{aligned}
(m-x-2) & =(m-2 x)+(x-2) \\
& \leq(x-2) \exp (m-2 x),
\end{aligned}
$$

with equality if and only if $x=\frac{m}{2}$. Then $(2-x)+(m-x-2) \exp (2 x-m) \leq 0$ with equality if and only if $x=\frac{m}{2}$. Let $f(x)=(x-1) \exp (n-x)+(m-x-$ $1) \exp (n-m+x)$ and $m \geq 8$ and $3 \leq x \leq \frac{m}{2}$. Then

$$
f^{\prime}(x)=\exp (n-x)((2-x)+(n-m-2) \exp (2 x-m)) \leq 0,
$$

with equality if and only if $x=\frac{m}{2}$. Let $m=n_{i}+n_{j}$ then

$$
f\left(n_{i}\right)<f\left(n_{i}-1\right)
$$

This implies that

$\left(n_{i}-1\right) \exp \left(n-n_{i}\right)+\left(n_{j}-1\right) \exp \left(n-n_{j}\right)<\left(n_{i}-2\right) \exp \left(n-n_{i}+1\right)+n_{j} \exp \left(n-n_{j}-1\right)$.

Hence, replacing any pair $\left(n_{i}, n_{j}\right)$ by $\left(n_{i}-1, n_{j}+1\right)$ in (??), the sum increases.

The next result relates the $L E E$ of a connected graph $\mathcal{G}$, when an edge is added, $\mathcal{G}+e$, and the $L E E$ of the original graph.

Lemma 5. [?] Let $\mathcal{G}$ be a connected graph on $n$ vertices non isomorphic to the complete graph. Then

$$
\operatorname{LEE}(\mathcal{G})<\operatorname{LEE}(\mathcal{G}+e)
$$


Now we prove the main result of this section.

Theorem 6. Let $r \geq 3$. Let $\mathcal{G} \in \mathcal{B}_{n, \chi}^{r}$. Then

$$
\operatorname{LEE}(\mathcal{G}) \leq b(n, \chi, r)
$$

where

$b(n, \chi, r)=1+(\chi-1) \exp (n)+(r-1)(\chi-1) \exp (n-r)+(n-r \chi+r-1) \exp (r \chi-r)$,

with equality in (??) if and only if $\mathcal{G} \cong \mathcal{K}_{r} \underbrace{, \ldots, r}_{\chi-1}, \underbrace{n-r \chi+r}_{1}$.

Proof. Let $\mathcal{G} \in \mathcal{B}_{n, \chi}^{r}$ then $2 \leq \chi \leq \frac{n}{r}$. Let $\mathcal{G} \in \mathcal{B}_{n, \chi}^{r}$ be the graph that has the largest Estrada index among all the graphs $\mathcal{H} \in \mathcal{B}_{n, \chi}^{r}$. Then $\chi(\mathcal{G})=\chi$. Thus, let us consider the partition of $V(\mathcal{G})$ into $\chi$ color classes, say $V_{1}, V_{2}, \ldots, V_{\chi}$. From Lemma ??, each vertex in $V_{i}$ is adjacent to all vertices in $V_{j}$ for any $1 \leq i<j \leq \chi$. Then, $\mathcal{G} \cong \mathcal{K}_{n_{1}, n_{2}, \ldots, n_{\chi}}$, where $n_{i}=\left|V_{i}\right|$ for $1 \leq i \leq \chi$. Without loss of generality, we can assume that $r \leq n_{1} \leq n_{2} \leq$ $\cdots \leq n_{\chi}$. It is clear that

$$
\begin{aligned}
\operatorname{Sp}(\overline{\mathcal{G}}) & =S p\left(\mathcal{K}_{n_{1}} \cup \mathcal{K}_{n_{2}} \cup \cdots \cup \mathcal{K}_{n_{\chi}}\right) \\
& =(\underbrace{n_{\chi}, \ldots, n_{\chi}}_{n_{\chi}-1}, \ldots, \underbrace{n_{1}, \ldots, n_{1}}_{n_{1}-1}, \underbrace{0, \ldots, 0}_{\chi}) .
\end{aligned}
$$

Therefore,

$$
S p(\mathcal{G})=(\underbrace{n, \ldots, n}_{\chi-1}, \underbrace{n-n_{1}, \ldots, n-n_{1}}_{n_{1}-1}, \ldots, \underbrace{n-n_{\chi}, \ldots, n-n_{\chi}}_{n_{\chi}-1}, 0) .
$$

Thus, we obtain

$$
L E E(\mathcal{G})=(\chi-1) \exp (n)+1+\sum_{i=1}^{\chi}\left(n_{i}-1\right) \exp \left(n-n_{i}\right)
$$

with $n=\sum_{i=1}^{\chi} n_{i}$.

Suppose $n_{\chi-1} \geq r+1$. Either for $\chi=2$ or $\chi \geq 3$, a new graph $\mathcal{H}$ such that $\mathcal{H} \cong \mathcal{K}_{n_{1}-1, n_{2}+1}$ in the former and $\mathcal{H} \cong \mathcal{K}_{n_{1}, \ldots, n_{\chi-1}-1, n_{\chi}+1}$ in the latter, can be 
constructed. In both cases $\mathcal{H} \in \mathcal{B}_{n, \chi}^{r}$. By Lemma ??, $\operatorname{LEE}(\mathcal{G})<L E E(\mathcal{H})$ which is a contradiction to the maximality of $\mathcal{G}$ in $\mathcal{B}_{n, \chi}^{r}$. Therefore,

$L E E(\mathcal{G}) \leq 1+(\chi-1) \exp (n)+(r-1)(\chi-1) \exp (n-r)+(n-r \chi+r-1) \exp (r \chi-r)$,

with equality if and only if $\mathcal{G} \cong \mathcal{K}_{r} \underbrace{, \ldots, r}_{\chi-1}, \underbrace{n-r \chi+r}_{1}$.

Using Theorem ?? and the fact that $\mathcal{G}$ is a bipartite graph if and only if $\chi(\mathcal{G})=2$, see [?], the following corollary is an immediate consequence.

Corollary 7. Let $\mathcal{G}$ be a bipartite graph on $n$ vertices with color classes of order greater than or equal to $r \geq 3$. Then

$$
L E E(\mathcal{G}) \leq 1+\exp (n)+(r-1) \exp (n-r)+(n-r-1) \exp (r),
$$

where equality holds in (??) if and only if $\mathcal{G} \cong \mathcal{K}_{r, n-r}$.

\section{An upper bound for the Estrada Index of the complement of a graph in $\mathcal{B}_{n, \chi}^{r}$}

In this section, we present an upper bound for the Estrada index of the complement of a graph in the family of graphs $\mathcal{B}_{n, \chi}^{r}$. At this point, we recall that if $\mu_{1} \geq \mu_{2} \geq \cdots \geq \mu_{n-1} \geq \mu_{n}=0$ are the Laplacian eigenvalues of $\mathcal{G}$, then, $0 \leq n-\mu_{1} \leq \cdots \leq n-\mu_{n-1}$ are the Laplacian eigenvalues of $\overline{\mathcal{G}}$. Taking into account that the exponential function is a real increasing function, we can say that for a graph $H, L E E(H)$ increases while the Laplacian eigenvalues of $H$ increase and, in consequence while the Laplacian eigenvalues of $\bar{H}$ diminish.

If $\mathcal{G} \in \mathcal{B}_{n, \chi}^{r}$ and if it is such that $\overline{\mathcal{G}}$ has its eigenvalues as the largest possible, then $\mathcal{G}$ has its eigenvalues as the smallest possible.

For instance, if $\mathcal{G}$ has the possible maximum quantity of Laplacian eigenvalues equals to zero, then $\overline{\mathcal{G}}$ has the possible maximum quantity of Laplacian eigenvalues equals to $n$. But, this occurs when $\mathcal{G}$ has the possible maximum number of connected components. The next result gives an upper bound for the Estrada index of the complement of a graph in the family $\mathcal{B}_{n, 2}^{r}$. 
Theorem 8. Let $\mathcal{G} \in \mathcal{B}_{n, 2}^{r}$ then

$$
\begin{aligned}
\operatorname{LEE}(\overline{\mathcal{G}}) \leq & \left(r_{1}-1\right)(\exp (n-2)+\exp (n))+\exp (n-1-s)+ \\
& (s-1) \exp (n-1)+1 \\
= & \left(r_{1}-1\right)(\exp (n-2)+\exp (n))+\exp \left(2 r_{1}-2\right)+ \\
& \left(n-2 r_{1}\right) \exp (n-1)+1,
\end{aligned}
$$

where $n=2 r_{1}+s-1$, with $r_{1} \geq r$ and $s \geq r-r_{1}+1$.

Proof. Suppose that the number of elements of the color classes in $\mathcal{G}$ are $r_{1}$ and $r_{2}$. Without loss of generality suppose that $r \leq r_{1} \leq r_{2}$. Suppose that $r_{2}=r_{1}-1+s$. By increasing the number of edges in $\overline{\mathcal{G}}$ the Estrada index increases, then $\overline{\mathcal{G}}=\mathcal{K}_{r_{1}} \vee \mathcal{K}_{r_{2}}-\left[M \cup \mathcal{K}_{1, s}\right]$, where $M$ is a matching with $r_{1}-1$ edges. In this case, $\mathcal{G}=\left(r_{1}-1\right) \mathcal{K}_{2} \cup \mathcal{K}_{1, s}$. In consequence $\operatorname{Sp}(\overline{\mathcal{G}})=(n-2, n, n-1-s, n-1,0)$. Thus, the inequality in Theorem ?? holds .

Remark 9. Taking into account that the right hand side of the inequality in Theorem ?? is an increasing function of $r_{1}$ which is at most $\frac{n}{2}$ (as $r \leq r_{1} \leq$ $\frac{n}{2} \leq r_{2}$, otherwise we obtain a contradiction), from the same inequality we have:

$$
\operatorname{LEE}(\overline{\mathcal{G}}) \leq 1+\left(\frac{n}{2}-1\right) \exp (n)+\frac{n}{2} \exp (n-2) .
$$

Now, taking into account [? , Corollary 13], the following Nordhaus-Gaddum type inequality, when $\mathcal{G}$ is bipartite can be obtained.

Corollary 10. Let $\mathcal{G}$ be a bipartite graph on $n$ vertices with color classes of order greater than or equal to $r \geq 3$. Then, for $n \neq 6$

$$
L E E(\mathcal{G})+L E E(\overline{\mathcal{G}}) \leq 2+\frac{n}{2} \exp (n)+\left(1+\frac{n}{2}\right) \exp (n-2)+(n-3) \exp (2) .
$$

\section{Estrada Index and Connectivity (vertex and edge)}

In this section, it is given an upper bound for the Estrada index of the line graph of undirected simple connected graphs whose vertex (and edge) connectivity is less than or equal to a certain positive $k$. The equality cases are discussed. Recall that the vertex connectivity (or just connectivity) of a graph $\mathcal{G}$, denoted by $\kappa(\mathcal{G})$, is the smallest number of vertices of $\mathcal{G}$ whose 
removals results in a disconnect graph. It is conventional to define $\kappa\left(\mathcal{K}_{n}\right)=$ $n-1$. Moreover, the edge connectivity $\epsilon(\mathcal{G})$, is the smallest number of edges whose removals results in a disconnected graph. Always $\kappa(\mathcal{G}) \leq \epsilon(\mathcal{G}) \leq \delta((\mathcal{G})$, see [?, Theorem 5.1].

For $n$ and $k$ fixed positive integers with $k \leq n-1$, the signless Laplacian eigenvalues of the family of join graphs $\mathcal{K}_{k} \vee\left(\mathcal{K}_{i} \cup \mathcal{K}_{n-k-i}\right)$ with $1 \leq i \leq\left\lfloor\frac{n-k}{2}\right\rfloor$ are identified in the following result.

Lemma 11. [?] The signless Laplacian eigenvalues of $\mathcal{K}_{k} \vee\left(\mathcal{K}_{i} \cup \mathcal{K}_{n-k-i}\right)$ are

$$
\begin{aligned}
& n+\frac{k}{2}-2+\frac{1}{2} \sqrt{(2 n-k)^{2}+16 i(k-n+i)}, \\
& n+\frac{k}{2}-2-\frac{1}{2} \sqrt{(2 n-k)^{2}+16 i(k-n+i)},
\end{aligned}
$$

with multiplicity 1 and,

$$
n-2, k+i-2, n-i-2,
$$

with multiplicity $k, i-1$ and $n-k-i-1$, respectively.

The following result relates the signless Laplacian eigenvalues of a graph $\mathcal{G}$ to the eigenvalues of its line graph.

Lemma 12. [? ? ] Let $\mathcal{G}$ be a graph of order $n$ with $m \geq 1$ edges. Let $q_{i}$ be the $i$-th greatest signless Laplacian eigenvalue of $\mathcal{G}$ and $\lambda_{i}(\mathcal{L}(\mathcal{G}))$ the $i$-th greatest eigenvalue of the line graph $\mathcal{L}(\mathcal{G})$ of $\mathcal{G}$. Then

$$
q_{i}=\lambda_{i}(\mathcal{L}(\mathcal{G}))+2,
$$

for $i=1,2, \ldots, k$, where $k=\min \{n, m\}$. In addition, if $m>n$, then $\lambda_{i}(\mathcal{L}(\mathcal{G}))=-2$ for $i \geq n+1$ and if $n>m$, then $q_{i}=0$ for $i \geq m+1$.

A direct consequence of Lemma ?? and Lemma ?? is the following.

Lemma 13. [?] The eigenvalues of the line graph of $\mathcal{K}_{k} \vee\left(\mathcal{K}_{i} \cup \mathcal{K}_{n-k-i}\right)$ are given by

$$
\begin{aligned}
& n+\frac{k}{2}-4+\frac{1}{2} \sqrt{(2 n-k)^{2}+16 i(k-n+i)}, \\
& n+\frac{k}{2}-4-\frac{1}{2} \sqrt{(2 n-k)^{2}+16 i(k-n+i)},
\end{aligned}
$$


with multiplicity 1 and,

$$
n-4, k+i-4, n-i-4,-2 \text {, }
$$

with multiplicity $k, i-1, n-k-i-1$ and $m-n$, respectively.

As a direct consequence of the dominating non-negative matrix property, see [? ], it is obtained below.

Lemma 14. [?] Let $\mathcal{G}$ be a connected graph then

$$
\lambda_{1}(\mathcal{L}(\mathcal{G}))<\lambda_{1}(\mathcal{L}(\mathcal{G}+e)) .
$$

A direct consequence from the Lemma ?? and Cauchy Interlacing Theorem, $[?$, Theorem 1] is the following result.

Theorem 15. Let $\mathcal{G}$ be a connected graph on $n$ vertices non-isomorphic to the complete graph. Then

$$
E E(\mathcal{L}(\mathcal{G}))<E E(\mathcal{L}(\mathcal{G}+e))
$$

Proof. By taking into account that the adjacency matrix of the line graph $\mathcal{L}(\mathcal{G})$ is a submatrix of the line graph $\mathcal{L}(\mathcal{G}+e)$. Since the exponential function is strictly increasing, the inequality below follows from the Cauchy Interlacing Theorem,

$$
E E(\mathcal{L}(\mathcal{G})) \leq E E(\mathcal{L}(\mathcal{G}+e))
$$

The strict inequality in (??) results from Lemma ??.

An immediate consequence of the above result is as follows.

Corollary 16. Among all the graphs on $n$ vertices, the complete graph $\mathcal{K}_{n}$ has the largest Estrada index of its line graph.

In the next lemma the number of edges of the graph $\mathcal{K}_{k} \vee\left(\mathcal{K}_{i} \cup \mathcal{K}_{n-k-i}\right)$, $1 \leq i \leq\left\lfloor\frac{n-k}{2}\right\rfloor$ is given.

Lemma 17. [? ] The number of edges of the graph $\mathcal{K}_{k} \vee\left(\mathcal{K}_{i} \cup \mathcal{K}_{n-k-i}\right)$, $1 \leq i \leq\left\lfloor\frac{n-k}{2}\right\rfloor$ is given by

$$
\frac{2 i^{2}-2 i(n-k)+n^{2}-n}{2} .
$$


Using the derivative of a function the next result is obtained.

Lemma 18. Let $f$ be a continuous function in the interval $[a, b]$. Suppose $f$ has negative derivative in the interval $(a, b)$. Suppose $f(b)>0$. Then

$$
h(x)=\exp (f(x))+\exp (-f(x))
$$

is a strictly decreasing function in $[a, b]$.

Proof. Let $f$ be a continuous function in $[a, b]$ with negative derivative in $(a, b)$, then $f$ is strictly decreasing in $[a, b]$. Since $f(b)>0$ then $f(x)>0$ for all $x \in[a, b]$.

Since $h(x)=\exp (f(x))+\exp (-f(x))$ then $h$ is a continuous function in $[a, b]$ and differentiable in $(a, b)$. Moreover

$$
h^{\prime}(x)=f^{\prime}(x)\left[\frac{\exp (2 f(x))-1}{\exp (f(x))}\right] .
$$

Since $f^{\prime}(x)<0$ for all $x \in(a, b)$ then $h(x)<0$ for all $x \in(a, b)$. Hence, $h$ is strictly decreasing in $[a, b]$.

The eigenvalues of the line graph of a regular graph can be found in [? ].

Lemma 19. [?] Let $\lambda_{1}, \lambda_{2}, \ldots, \lambda_{n}$ be the eigenvalues of a regular graph $\mathcal{G}$ of order $n$ and degree $r$, then the eigenvalues of $\mathcal{L}(\mathcal{G})$ are $\lambda_{i}+r-2, i=1,2, \ldots, n$ and -2 , with multiplicity $\frac{n(r-2)}{2}$.

Consider now $\mathcal{F}_{n}$ the family of the simple undirected connected graphs on $n$ vertices. Let

$$
\mathcal{V}_{n}^{k}=\left\{\mathcal{G} \in \mathcal{F}_{n}: \kappa(\mathcal{G}) \leq k\right\} .
$$

The next theorem gives an upper bound for the Estrada index of the line graph of a graph. The equality case is discussed.

Theorem 20. Let $\mathcal{G} \in \mathcal{V}_{n}^{k}$. Then

$$
E E(\mathcal{L}(\mathcal{G})) \leq b(n, k)
$$

where

$$
\begin{aligned}
b(n, k) & =k \exp (n-4)+(n-k-2) \exp (n-5)+\frac{n^{2}-5 n+2 k+2}{2} \exp (-2) \\
& +\exp \left(n-\frac{k}{2}-4+\frac{1}{2} \sqrt{(2 n-k)^{2}-16(k-n+1)}\right) \\
& +\exp \left(n-\frac{k}{2}-4-\frac{1}{2} \sqrt{(2 n-k)^{2}-16(k-n+1)}\right),
\end{aligned}
$$

with equality in (??) if and only if $\mathcal{G} \cong \mathcal{K}_{k} \vee\left(\mathcal{K}_{1} \cup \mathcal{K}_{n-k-1}\right)$. 
Proof. Let $\mathcal{G} \in \mathcal{V}_{n}^{k}$. We first consider $k=n-1$. From Corollary ??, $E E(\mathcal{L}(\mathcal{G})) \leq E E\left(\mathcal{L}\left(\mathcal{K}_{n}\right)\right)$ with equality if and only if $\mathcal{G} \cong \mathcal{K}_{n}$. It is well known that

$$
\operatorname{Sp}\left(\mathcal{K}_{n}\right)=\left\{n-1,(-1)^{[n-1]}\right\} .
$$

From Lemma ??, we have

$$
S p\left(\mathcal{L}\left(\mathcal{K}_{n}\right)\right)=\left\{2 n-4,(n-4)^{[n-1]},(-2)^{\frac{n(n-3)}{2}}\right\} .
$$

Thus,

$$
E E\left(\mathcal{L}\left(\mathcal{K}_{n}\right)\right)=\exp (2 n-4)+(n-1) \exp (n-4)+\frac{n(n-3)}{2} \exp (-2) .
$$

Then the result is true for $k=n-1$. Consider $1 \leq k \leq n-2$. Let $\mathcal{G} \in \mathcal{V}_{n}^{k}$ such that $\mathcal{L}(\mathcal{G})$ has the largest Estrada index among all the graphs $\mathcal{L}(\mathcal{H})$ with $\mathcal{H} \in \mathcal{V}_{n}^{k}$. Let $U \subset V(\mathcal{G})$ such that $\mathcal{G}-U$ is a disconnected graph. Let $\mathcal{Y}_{1}, \mathcal{Y}_{2}, \ldots, \mathcal{Y}_{r}$, be the connected components of $\mathcal{G}-U$. We claim that $r=2$. If $r>2$ then we can construct a graph $\mathcal{H}=\mathcal{G}+e$, where $e$ is an edge connecting a vertex in $\mathcal{Y}_{1}$ to a vertex in $\mathcal{Y}_{2}$. Clearly, $\mathcal{H} \in \mathcal{V}_{n}^{k}$. By Theorem ??, $E E(\mathcal{L}(\mathcal{G}))<E E(\mathcal{L}(\mathcal{H}))$, which is a contradiction to the maximality of $\mathcal{G}$ in $\mathcal{V}_{n}^{k}$. Therefore $r=2$, and $\mathcal{G}-U=\mathcal{Y}_{1} \cup \mathcal{Y}_{2}$. We have $|U| \leq k$. Now, we claim that $|U|=k$. Suppose $|U|<k$. We construct a graph $\mathcal{H}=\mathcal{G}+e$ where $e$ is an edge joining a vertex $u \in \mathcal{Y}_{1}$ with a vertex $v \in \mathcal{Y}_{2}$. We see that $\mathcal{H}-U$ is a connected graph and the deletion of the vertex $u$ disconnects $\mathcal{H}-U$. This tell us that $\mathcal{H} \in \mathcal{V}_{n}^{k}$. By Theorem ??, $E E(\mathcal{L}(\mathcal{G}))<E E(\mathcal{L}(\mathcal{H}))$, which is a contradiction. Then, $|U|=k$. Therefore, $\mathcal{G}-U=\mathcal{Y}_{1} \cup \mathcal{Y}_{2}$ and $|U|=k$. Let $\left|\mathcal{Y}_{1}\right|=i$ then $\left|\mathcal{Y}_{2}\right|=n-k-i$.

We claim

$$
\mathcal{G} \cong \mathcal{K}_{k} \vee\left(\mathcal{K}_{i} \cup \mathcal{K}_{n-k-i}\right)
$$

for some $1 \leq i \leq\left\lfloor\frac{n-k}{2}\right\rfloor$. On the contrary, suppose that $\mathcal{Y}_{3}$ is the induced subgraph of $\mathcal{G}$ obtained from the vertices in $U$, then there exists an edge in the set

$$
\left[E\left(\overline{\mathcal{Y}_{1}} \vee \overline{\mathcal{Y}_{3}}\right) \cup E\left(\overline{\mathcal{Y}_{2}} \vee \overline{\mathcal{Y}_{3}}\right)\right]-E(\mathcal{G})
$$

Therefore, it is possible to construct a new graph $\mathcal{H}=\mathcal{G}+e$. Clearly, $\mathcal{H} \in \mathcal{V}_{n}^{k}$. By Theorem ??, $E E(\mathcal{L}(\mathcal{G}))<E E(\mathcal{L}(\mathcal{H}))$ which is a contradiction.

Until this point, we have proved that

$$
E E(\mathcal{L}(\mathcal{G})) \leq E E\left(\mathcal{L}\left(\mathcal{K}_{k} \vee\left(\mathcal{K}_{i} \cup \mathcal{K}_{n-k-i}\right)\right)\right)
$$


for all $\mathcal{G} \in \mathcal{V}_{n}^{k}$. Now we search the value of $i$ for which $E E\left(\mathcal{L}\left(\mathcal{K}_{k} \vee\left(\mathcal{K}_{i} \cup\right.\right.\right.$ $\left.\left.\mathcal{K}_{n-k-i}\right)\right)$ ) is maximum. From Lemma ?? and Lemma ??,

$$
\begin{aligned}
E E\left(\mathcal{L}\left(\mathcal{K}_{k} \vee\left(\mathcal{K}_{i} \cup \mathcal{K}_{n-k-i}\right)\right)\right) & =(i-1) \exp (k+i-4)+(n-k-i-1) \exp (n-i-4) \\
& +k \exp (n-4)+\frac{2 i^{2}-2(n-k) i+n^{2}-3 n}{2} \exp (-2) \\
& +\exp \left(n+\frac{k}{2}-4+\frac{1}{2} \sqrt{(2 n-k)^{2}+16 i(k-n+i)}\right) \\
& +\exp \left(n+\frac{k}{2}-4-\frac{1}{2} \sqrt{(2 n-k)^{2}+16 i(k-n+i)}\right) .
\end{aligned}
$$

Define

$$
f(x)=g(x)+h(x)
$$

where,

$$
\begin{aligned}
g(x) & =(x-1) \exp (k+x-4) \\
& +(n-k-x-1) \exp (n-x-4) \\
& +\frac{2 x^{2}-2(n-k) x+n^{2}-3 n}{2} \exp (-2)
\end{aligned}
$$

and

$$
h(x)=\exp \left(n+\frac{k}{2}-4+p(x)\right)+\exp \left(n+\frac{k}{2}-4-p(x)\right)
$$

where

$$
p(x)=\frac{1}{2} \sqrt{(2 n-k)^{2}+16 x(k-n+x)} .
$$

Thus,

$g^{\prime}(x)=x \exp (k+x-4)-(n-k-x) \exp (n-x-4)+(2 x-(n-k)) \exp (-2)$.

Since $x \exp (k+x-4) \leq(n-k-x) \exp (n-x-4)$ for $x \in\left[1, \frac{n-k}{2}\right]$ with equality only for $x=\frac{n-k}{2}$, then $g^{\prime}(x) \leq 0$ with equality for $x=\frac{n-k}{2}$. Thus, $g(x)$ is strictly decreasing in $\left[1, \frac{n-k}{2}\right]$. Since $p(x)$ in (??) is continuous in $\left[1, \frac{n-k}{2}\right]$ with negative derivative in $\left(1, \frac{n-k}{2}\right)$ and $p\left(\frac{n-k}{2}\right)=\frac{\sqrt{4 n k-3 k^{2}}}{2}>0$, by Lemma ??, $h(x)$ is strictly decreasing in $\left[1, \frac{n-k}{2}\right]$. Hence, $f(x)$ is strictly decreasing in this interval. 
Therefore

$$
E E(\mathcal{L}(\mathcal{G})) \leq E E\left(\mathcal{L}\left(\mathcal{K}_{k} \vee\left(\mathcal{K}_{1} \cup \mathcal{K}_{n-k-1}\right)\right)\right)
$$

for all $\mathcal{G} \in \mathcal{V}_{n}^{k}$ with equality if and only if $\mathcal{G} \cong \mathcal{K}_{k} \vee\left(\mathcal{K}_{1} \cup \mathcal{K}_{n-k-1}\right)$.

In graphs of communication or transportation networks, the edge connectivity is an important measure of reliability. The minimum degree of a graph $\mathcal{G}$, will be denoted by $\delta(\mathcal{G})$.

Let

$$
\Omega_{n}^{k}=\left\{\mathcal{G} \in \mathcal{F}_{n}: \epsilon(\mathcal{G}) \leq k\right\},
$$

and

$$
\Delta_{n}^{k}=\left\{\mathcal{G} \in \mathcal{F}_{n}: \delta(\mathcal{G}) \leq k\right\} .
$$

Recall that $\kappa(\mathcal{G}) \leq \epsilon(\mathcal{G}) \leq \delta(\mathcal{G})$, [? ? ], then $\Omega_{n}^{k} \subseteq \mathcal{V}_{n}^{k}$ and $\Delta_{n}^{k} \subseteq \mathcal{V}_{n}^{k}$. Thus for $\mathcal{G} \in \Omega_{n}^{k}$ or $\mathcal{G} \in \Delta_{n}^{k}$ we have $\mathcal{G} \in \mathcal{V}_{n}^{k}$. Since $\mathcal{K}_{k} \vee\left(\mathcal{K}_{1} \cup \mathcal{K}_{n-k-1}\right) \in \Omega_{n}^{k}$ has minimum degree $k$ then, as a consequence of the Theorems ?? and ??, the following result holds.

Corollary 21. Let $\mathcal{G} \in \Omega_{n}^{k} \cup \Delta_{n}^{k}$. Then

$$
E E(\mathcal{L}(\mathcal{G})) \leq b(n, k)
$$

where

$$
\begin{aligned}
b(n, k) & =k \exp (n-4)+(n-k-2) \exp (n-5)+\frac{n^{2}-5 n+2 k+2}{2} \exp (-2) \\
& +\exp \left(n-\frac{k}{2}-4+\frac{1}{2} \sqrt{(2 n-k)^{2}-16(k-n+1)}\right) \\
& +\exp \left(n-\frac{k}{2}-4-\frac{1}{2} \sqrt{(2 n-k)^{2}-16(k-n+1)}\right),
\end{aligned}
$$

with equality in (??) if and only if $\mathcal{G} \cong \mathcal{K}_{k} \vee\left(\mathcal{K}_{1} \cup \mathcal{K}_{n-k-1}\right)$.

\section{Signless Laplacian Estrada Index and Connectivity}

The aim of this section is to establish a result about graphs with maximal signless Laplacian Estrada index.

Lemma 22. [? ] Let $\mathcal{G}$ a connected graph on $n$ vertices nonisomorphic to the complete graph. Then

$$
S L E E(\mathcal{G})<S L E E(\mathcal{G}+e) .
$$


The following is an immediate consequence.

Corollary 23. Among all graphs on $n$ vertices, the complete graph $\mathcal{K}_{n}$ has the largest signless Laplacian Estrada index.

The next theorem gives an upper bound for the signless Laplacian Estrada index of a graph. The equality case is discussed.

Theorem 24. Let $\mathcal{G} \in \mathcal{V}_{n}^{k}$. Then

$$
\operatorname{SLEE}(\mathcal{G}) \leq c(n, k)
$$

where

$$
\begin{aligned}
c(n, k) & =k \exp (n-2)+(n-k-2) \exp (n-3) \\
& +\exp \left(n-\frac{k}{2}-2+\frac{1}{2} \sqrt{(2 n-k)^{2}-16(k-n+1)}\right) \\
& +\exp \left(n-\frac{k}{2}-2-\frac{1}{2} \sqrt{(2 n-k)^{2}-16(k-n+1)}\right),
\end{aligned}
$$

with equality in (??) if and only if $\mathcal{G} \cong \mathcal{K}_{k} \vee\left(\mathcal{K}_{1} \cup \mathcal{K}_{n-k-1}\right)$.

Proof. Let $\mathcal{G} \in \mathcal{V}_{n}^{k}$. We first consider $k=n-1$. From Corollary ??, $S L E E(\mathcal{G}) \leq S \operatorname{LEE}\left(\mathcal{K}_{n}\right)$ with equality if and only if $\mathcal{G} \cong \mathcal{K}_{n}$. Moreover,

$$
S L E E\left(\mathcal{K}_{n}\right)=\exp (2 n-2)+(n-1) \exp (n-2) \text {. }
$$

Then the result is true for $k=n-1$. Let now $1 \leq k \leq n-2$. Using Lemma ?? and the same techniques from Theorem ??, we can conclude that

$$
\operatorname{SLEE}(\mathcal{G}) \leq S L E E\left(\mathcal{K}_{k} \vee\left(\mathcal{K}_{i} \cup \mathcal{K}_{n-k-i}\right)\right)
$$

for all $\mathcal{G} \in \mathcal{V}_{n}^{k}$. We now search for a value of $i$ for which $S L E E\left(\mathcal{K}_{k} \vee\left(\mathcal{K}_{i} \cup\right.\right.$ $\left.\mathcal{K}_{n-k-i}\right)$ ) is maximum. From Lemma ??, we obtain

$$
\begin{aligned}
S L E E\left(\mathcal{K}_{k} \vee\left(\mathcal{K}_{i} \cup \mathcal{K}_{n-k-i}\right)\right) & =k \exp (n-2) \\
& +(i-1) \exp (k+i-2)+(n-k-i-1) \exp (n-i-2) \\
& +\exp \left(n+\frac{k}{2}-2+\frac{1}{2} \sqrt{(2 n-k)^{2}+16 i(k-n+i)}\right) \\
& +\exp \left(n+\frac{k}{2}-2-\frac{1}{2} \sqrt{(2 n-k)^{2}+16 i(k-n+i)}\right) .
\end{aligned}
$$


Define the function

$$
f(x)=g(x)+h(x)
$$

where

$$
g(x)=(x-1) \exp (k+x-2)+(n-k-x-1) \exp (n-x-2)
$$

and

$$
h(x)=\exp \left(n+\frac{k}{2}-2+p(x)\right)+\exp \left(n+\frac{k}{2}-2-p(x)\right),
$$

where $p(x)$ is defined in (??).

Thus

$$
g^{\prime}(x)=x \exp (k+x-2)-(n-k-x) \exp (n-x-2) .
$$

Since $x \exp (k+x-2) \leq(n-k-x) \exp (n-x-2)$ for $x \in\left[1, \frac{n-k}{2}\right]$ with equality only for $x=\frac{n-k}{2}$, then, $g^{\prime}(x) \leq 0$ with equality only for $x=\frac{n-k}{2}$. Thus, $g(x)$ is strictly decreasing in $\left[1, \frac{n-k}{2}\right]$. Using a strategy similar to the proof of Theorem ?? using the function $p(x)$, we conclude that, $h(x)$ is strictly decreasing in $\left[1, \frac{n-k}{2}\right]$. Hence, $f(x)$ is strictly decreasing in this interval. Consequently

$$
\operatorname{SLEE}(\mathcal{G}) \leq S L E E\left(\mathcal{K}_{k} \vee\left(\mathcal{K}_{1} \cup \mathcal{K}_{n-k-1}\right)\right)
$$

for all $\mathcal{G} \in \mathcal{V}_{n}^{k}$ with equality if and only if $\mathcal{G} \cong \mathcal{K}_{k} \vee\left(\mathcal{K}_{1} \cup \mathcal{K}_{n-k-1}\right)$.

Corollary 25. Let $\mathcal{G} \in \Omega_{n}^{k} \cup \Delta_{n}^{k}$. Then

$$
\operatorname{SLEE}(\mathcal{G}) \leq c(n, k)
$$

where

$$
\begin{aligned}
c(n, k) & =k \exp (n-2)+(n-k-2) \exp (n-3) \\
& +\exp \left(n-\frac{k}{2}-2+\frac{1}{2} \sqrt{(2 n-k)^{2}-16(k-n+1)}\right) \\
& +\exp \left(n-\frac{k}{2}-2-\frac{1}{2} \sqrt{(2 n-k)^{2}-16(k-n+1)}\right),
\end{aligned}
$$

with equality in (??) if and only if $\mathcal{G} \cong \mathcal{K}_{k} \vee\left(\mathcal{K}_{1} \cup \mathcal{K}_{n-k-1}\right)$. 


\section{Comparisons and final remarks}

In this section, we present some computational experiments to compare our new upper bounds with real values of the Estrada indices for certain connected graphs. We compare the estimates values obtained by Theorems ??, ??, ??, and Remark ?? with the approximate Estrada indices of each graph in the table. From the results obtained in Theorems ?? and ??, we have $E E(\mathcal{L}(\mathcal{G})) \leq 1.888 .017,0$ and $\operatorname{SLEE}(\mathcal{G}) \leq 13.898 .082,6$, for each $\mathcal{G} \in \mathcal{V}_{10}^{3}$, respectively. From the results obtained in Theorem ?? and Remark ??, we have $\operatorname{LEE}(\mathcal{G}) \leq 24.341,2$ and $\operatorname{LEE}(\overline{\mathcal{G}}) \leq 103.011,6$, for each $\mathcal{G} \in \mathcal{B}_{10,2}^{3}$, respectively. Before presenting the comparative table we give the definitions of some graphs mentioned in it. Let $D$ be a set of positive, proper divisors of the integer $n>1$. Define the graph $I C G_{n}(D)$ to have vertex set $Z_{n}=$ $\{0,1, \ldots, n-1\}$ and edge set

$$
E\left(I C G_{n}(D)\right)=\left\{i j: i, j \in Z_{n}, \operatorname{gcd}(i-j, n) \in D\right\}
$$

see [? ]. Additionally, we define some non usual class of graphs which appear here in a number of conjectures. Namely, a lollipop $L o l_{n, g}$ is a graph obtained from a cycle on $g$ vertices by attaching a pendant path on $n-g$ vertices to one of its vertices. A turnip $T u_{n, g}$ is a graph obtained from a cycle on $g$ vertices by attaching $n-g$ pendant edges to one of its vertices. A kite $K i_{n, w}$ is a graph obtained from a clique on $w$ vertices by attaching a pendant path on $n-w$ vertices to one of its vertices. A bag $B a g_{p, q}$ is a graph obtained from a complete graph $\mathcal{K}_{p}$ by replacing an edge $u v$ by a path $\mathcal{P}_{q}$. A bug $B u g_{p, q_{1}, q_{2}}$ is a graph obtained from a complete graph $\mathcal{K}_{p}$ by deleting an edge $u v$ and attaching paths $\mathcal{P}_{q_{1}}$ and $\mathcal{P}_{q_{2}}$ at $u$ and $v$, respectively. A complete split graph $S K_{n, \alpha}$ is a graph obtained from an empty graph on $\alpha$ vertices and a clique on $n-\alpha$ vertices by adding all edges between them. 

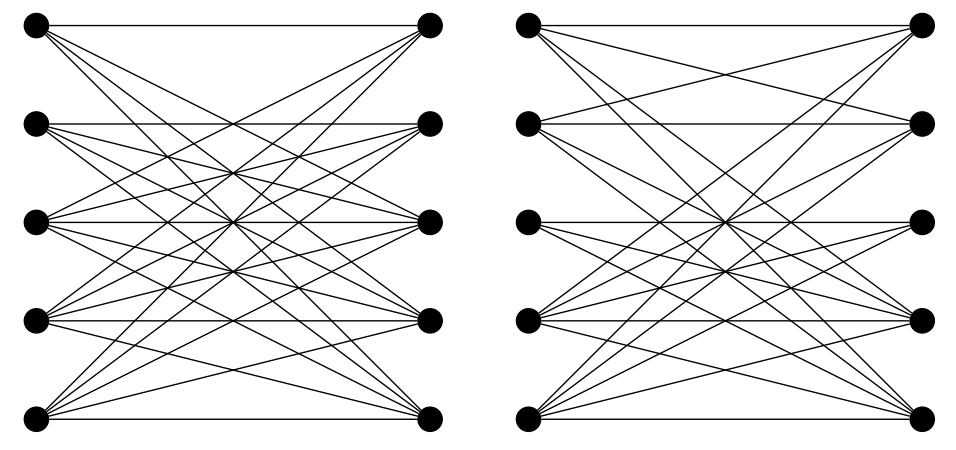

Figure 1: The graphs $\mathcal{L}_{1}$ and $\mathcal{L}_{2}$, respectively

\begin{tabular}{llllll}
\hline $\operatorname{Graph}(\mathcal{G})$ & $E E(\mathcal{L}(\mathcal{G}))$ & $S L E E(\mathcal{G})$ & $\operatorname{Graph}(\mathcal{G})$ & $L E E(\mathcal{G})$ & $L E E(\overline{\mathcal{G}})$ \\
\hline $\mathcal{P}_{10}$ & 19,0 & 141,7 & $\mathcal{P}_{10}$ & 141,7 & $56.773,3$ \\
$\mathcal{S}_{10}$ & $2.983,9$ & $22.049,2$ & $\mathcal{C}_{10}$ & 168,6 & $46.000,3$ \\
Petersen & $1.471,1$ & $10.859,8$ & $\mathcal{K}_{4,6}$ & $23.510,7$ & $2.182,9$ \\
Bugg, $_{8,2}$ & $132.407,1$ & $978.344,3$ & $\mathcal{K}_{3,7}$ & $\mathbf{2 4 . 3 4 1 , 2}$ & $6.622,0$ \\
$B a g_{9,3}$ & $1.005 .892,9$ & $7.432 .575,5$ & Lol $_{10,4}$ & 200,5 & $48.814,2$ \\
$K i_{10,8}$ & $189.579,1$ & $1.400 .790,7$ & Lol $_{10,6}$ & 194,0 & $47.833,2$ \\
$S K_{10,7}$ & $65.686,3$ & $485.345,6$ & $T u_{10,4}$ & $8.484,3$ & $55.599,9$ \\
$K i_{10,9}$ & $1.371 .006,2$ & $10.130 .414,6$ & $T u_{10,6}$ & $1.264,8$ & $52.031,3$ \\
$\mathcal{K}_{7,3}$ & $3.295,7$ & $24.341,2$ & $I C G_{10}(1)$ & $3.655,9$ & $4.988,6$ \\
$\mathcal{K}_{2} \vee\left(\mathcal{K}_{2} \cup \mathcal{K}_{6}\right)$ & $343.771,0$ & $2.540 .146,2$ & $I C G_{10}(1,5)$ & $23.214,8$ & $1.189,3$ \\
$\mathcal{K}_{3} \vee\left(\mathcal{K}_{1} \cup \mathcal{K}_{6}\right)$ & $\mathbf{1 . 8 8 8 . 0 1 7 , 0}$ & $\mathbf{1 3 . 8 9 8 . 0 8 2 , 6}$ & $\mathcal{L}_{1}$ & $12.680,4$ & $2.574,0$ \\
$B u g_{9,2,1}$ & $910.146,3$ & $6.725 .095,4$ & $\mathcal{L}_{2}$ & $7.571,3$ & $5.310,9$ \\
\hline
\end{tabular}

- In all our test cases, the upper bounds in Theorems ??, ??, and ?? were reached by the corresponding extreme graphs in each one of them, respectively. 


\section{Conclusions}

In summary, in this paper, it is studied a meaningful graph invariant (the Estrada index) on an important subclass of connected graphs. More concretely, we characterized the graph having the largest signless Laplacian Estrada index and Estrada index of its line graph among all graphs on $n$ vertices with connectivity less than or equal to a fixed number. Moreover, we determined the graph having the largest Laplacian Estrada index among all graphs on $n$ vertices and fixed chromatic number where each independent set has order no less than 3 . These extremal graphs obtained are quite concordant with the extremal ones for some other graph invariants, such as the spread [? ], Laplacian energy like [? ], energy [? ] and signless Laplacian energy [? ], and some others. For future research, it would be interesting to continue studying the Estrada index of graphs with some specific structures. Also, this class of graphs is very significant which deserves further investigation.

Acknowledgments: Enide Andrade was supported in part by the Portuguese Foundation for Science and Technology (FCT-Fundação para a Ciência e a Tecnologia), Portugal, through CIDMA - Center for Research and Development in Mathematics and Applications, within project UID/MAT/04106/2019. Eber Lenes was supported by the Dirección de Investigaciones of the Universidad del Sinú, Colombia. Exequiel Mallea-Zepeda was supported by Proyecto UTA-Mayor 4740-18, Universidad de Tarapacá, Chile. María Robbiano was partially supported by project VRIDT UCN N 20190403038.

The authors would like to thank the referee for his/her constructive suggestions and comments that improved the final version of this paper.

\section{References}

[1] E. Andrade, E. Lenes, E. Mallea-Zepeda, M. Robbiano, J. Rodríguez Z. Bounds for different spreads of line and total graphs, Lin. Algebra Appl. 579 (2019): 365-381.

[2] S. K. Ayyaswamya, S.Balachandrana, Y. B. Venkatakrishnana, I. Gutman. Signless Laplacian Estrada index, MATCH Commun. Math. Comput. Chem. 66 (2011): 785-794. 
[3] S. Azami, On Laplacian and signless Laplacian Estrada indices of graphs, MATCH Commun. Math. Comput. Chem. 74 (2015): 411-418.

[4] R. Carbó- Dorca, Smooth function topological structure descriptor based on graph-spectra, J. Math. Chem. 44 (2008): 373-378.

[5] X. Chen, Y. Hou, Some results on Laplacian Estrada index of graphs, MATCH Commun. Math. Comput. Chem. 73 (2015): 149-162.

[6] H. Deng, Slavko Radencović, I. Gutman, The Estrada Index. Applications of graph spectra, Zbornik radova 13 (21) Editors: Dragoš Cvetković and Ivan Gutman, Beograd: Mathematički istitute SANU.

[7] J. R. Dias, Structure/ formula informatics of isomeric sets of fluoranthenoid/fluorenoid and indacenoid hydrocarbons, J. Math Chem. 48 (2010) 313-329.

[8] R. Diestel, Graph Theory Second Edition. Springer (Graduate Texts in Mathematics; 173)

[9] Z. Du, B. Zhou, R. Xing, On maximum Estrada indices of graphs with given parameters, Linear Algebra and its Appl. 436 (2012): 3767-3772.

[10] E. Estrada, Characterization of $3 D$ molecular structure, Chem. Phys. Lett. 319 (2000): 713-718.

[11] E. Estrada, Topological structural classes of complex networks, Phys. Rev. E 75 (2007): 016103-1-12.

[12] E. Estrada, N. Hatano, Statistical-mechanical approach to subgraph centrality in complex networks, Chem. 106 (2007): 247-251.

[13] E. Estrada, Characterization of the amino acid contribution to the folding degree of proteins, Proteins 54 (2004) 727-737.

[14] E. Estrada, Atom bond connectivity and the energetic of branched alkanes, Chem. Phys. Lett. 463 (2008), 422-425.

[15] E. Estrada, Characterization of the folding degree of proteins, Bioinformatics 18 (2002): 697-704.

[16] E. Estrada, J. A. Rodríguez-Velázquez, Subgraph centrality in complex networks, Phys. Rev. E 71 (2005): 056103. 
[17] E. Estrada, J. A. Rodríguez-Velázquez, Spectral measures of bipartivity in complex networks, Phys. Rev. E 72 (2005): 046105.

[18] E. Estrada, J. A. Rodríguez-Velázquez, M. Randić, Atomic branching in molecules, Int. J. Quantum. Chem. 106 (2006): 823-832.

[19] G. H. Fath-Tabar, A. R. Ashrafi, I. Gutman, Note on Estrada and L-Estrada indices of graphs, Bull. Acad. Serbe Sci. Arts (Cl. Sci. Math. Natur.) 139 (2009): 1-16.

[20] R. Grone, R. Merris, V. S. Sunder, The Laplacian spectrum of a graph, SIAM J. Matrix Anal. Appl. 11 (1990): 218-238.

[21] I. Gutman, J. Durdević, Fluorranthene and its congeners- A graph theoretical study, MATCH Commun. Math. Comput. Chem. 60 (2008) 659-670.

[22] I. Gutman, J. Durdević, On $\pi$ - electron conjugation in the five-membered ring of fluoranthen-type benzenoid hydrocarbons, J. Serb. Chem. Soc. 74 (2009) 765-771.

[23] I. Gutman, J. Durdević, Cycles in dicyclopenta-derivatives of benzenoid hydrocarbons, MATCH Commun. Math. Comput. Chem. 65 (2011) 785-798.

[24] I. Gutman, L. Medina, Pamela Pizarro, M. Robbiano, Graphs with maximum Laplacian and signless Laplacian Estrada index, 339 (2016): 2664-2671.

[25] I. Gutman, H. Deng, S. Radenković, The Estrada index: An updated survey, in: D. Cvetković, I. Gutman (Eds.), Selected Topics on Applications of Graph Spectra, Math. Inst., Belgrade, (2011): 155-174.

[26] I. Gutman, E. Estrada, J. A. Rodríguez-Velázquez, On a graph-spectrumbased structure descriptor, Croat. Chem. Acta 80 (2007): 151-154.

[27] I. Gutman, M. Robbiano, D. M. Cardoso, E. A. Martins, L. Medina, O. Rojo, Energy of line graphs, Linear Algebra Appl. 433 (2010): 1312-1323.

[28] F. Harary, Graph Theory, Reading, MA: Addison-Wesley (1994).

[29] S. G. Hwang, Cauchy's Interlace Theorem for Eigenvalues of Hermitian Matrices, The American Mathematical Monthly, Vol. 111, n 2 (2004): 157-159.

[30] A. Ilić, Distance spectra and distance energy of integral circulant graphs, Linear Algebra and its Applications 433 (2010) 1005-1014. 
[31] A. Ilić, B. Zhou, Laplacian Estrada index of trees, MATCH Commun. Math. Comput. Chem. 63 (2010): 769-776.

[32] E. Lenes, E. Mallea, M. Robbiano, J. Rodríguez, On line graphs with maximum energy, Linear Algebra Appl. 545 (2018): 15-31.

[33] J. Li, J. Zhang, Characterizing graphs with maximal Laplacian Estrada index, Linear Algebra Appl. 465 (2015): 312-324.

[34] S. C. Li, H. B. Zhou, On the maximum and minimum Zagreb indices of graphs with connectivity at most $k$, Appl. Math. Lett. 23 (2010): 128-132.

[35] J. Li, J. M. Guo, W. C. Shiu, The normalized Laplacian Estrada index of a graph, Filomat 28 (2014): 365-371.

[36] J. Li, W. C. Shiu, A. Chang, On Laplacian Estrada index of a graph, Appl. Anal. Discr. Math. 3 (2009): 147-156.

[37] H. Minc, Nonnegative Matrices, A. Wiley- Interscience Publication, John Wiley and Sons, US (1988).

[38] B. Mohar, Some applications of Laplace eigenvalues of graphs, in: G. Hahn, G. Sabidussi (Eds.), Graph Symmetry, Kluwer Academic Publishers, Dordrecht, (1997): $22-275$.

[39] O. Rojo, E. Lenes, A sharp upper bound on the incidence energy of graphs in terms of connectivity, Linear Algebra and its Appl. 438 (2013) 1485-1493.

[40] H. Sachs, Uber Teiler, Faktoren und charakteristische Polynome von Graphen, Teil II, Wiss. Z. TH Ilmenau 13 (1967) 405-412.

[41] W. H. Wang, W. W. Xu, Graphs with the maximal Estrada indices, Linear Algebra Appl. 446 (2014): 314-328.

[42] H. Whitney, Congruent Graphs and the Connectivity of graphs, Amer. J. Math. 54 (1932): 150-168.

[43] B. Zhou, I. Gutman, More on the Laplacian Estrada index, Appl. Anal. Discr. Math. 3 (2009): 371-378.

[44] Z. Zhu, L. Tan, Z. Qiu, Tricyclic graph with maximal Estrada index, Discrete Appl. Math. 162 (2014) 364-372. 
[45] B. Zhu, On the Laplacian Estrada index of graphs, MATCH Commun. Math. Comput. Chem. 66 (2011): 769-776.

[46] B. Zhu, The Laplacian-energy like of graphs, Applied Mathematics Letters 24(2011) 1604-1607. 